\title{
A prospective, multisite study analyzing the percentage of urological cases that can be completely managed by telemedicine
}

\author{
Bruno Turcotte, MD'; Sophie Paquet, BSinf'; Anne-Sophie Blais, MD'; Annie-Claude Blouin, MD'; \\ Stéphane Bolduc, MD'; Michel Bureau, MD'; Yves Caumartin, MD'; Jonathan Cloutier, $M D^{\prime}$; \\ Marie-Pier Deschênes-Rompré, MD'; Thierry Dujardin, MD'; Yves Fradet, MD'; Louis Lacombe, MD'; \\ Katherine Moore, $M D^{\prime}$; Fannie Morin, MD'; Geneviève Nadeau, MD, MSc' ; David Simonyan, $P h D^{2}$; \\ Frédéric Soucy, MD'; Rabi Tiguert, MD'; Paul Toren, $M D^{\prime}$; Michele Lodde, $M D^{\prime}$; Frédéric Pouliot, MD, PhD'
}

'Division of Urology, Department of Surgery, CHU de Québec-Université Laval, Quebec City, QC, Canada; ${ }^{2}$ Clinical and Evaluative Research Platform, Research Centre, CHU de Québec-Université Laval, Quebec City, QC, Canada

Cite as: Turcotte B, Paquet S, Blais A-S, et al. A prospective, multisite study analyzing the percentage of urological cases that can be completely managed by telemedicine. Can Urol Assoc J 2020;14(10):319-21. http://dx.doi.org/10.5489/cuaj.6862

\section{Abstract}

Introduction: The COVID-19 pandemic has accelerated the development of telemedicine due to confinement measures. However, the percentage of outpatient urological cases that could be managed completely by telemedicine outside of the COVID-19 pandemic remains to be determined. We conducted a prospective, multisite study involving all urologists working in the region of Quebec City.

Methods: During the first four weeks of the regional confinement, 18 pediatric and adult urologists were asked to determine, after each telemedicine appointment, if it translated into a complete (CCM), incomplete (ICM), or suboptimal case management (SCM, adequate only in the context of the pandemic).

Results: A total of 1679 appointments representing all urological areas were registered. Overall, 67.6\% (95\% confidence interval [CI] $65.3 ; 69.8), 27.1 \%(25.0 ; 29.3)$, and $4.3 \%(3.5 ; 5.4)$ were reported as CCM, SCM, and ICM, respectively. The CCM ratio varied according to the reason for consultation, with cancer suspicion $(52.9 \%[42.9 ; 62.8])$ and pediatric reasons $(38.0 \%$ [30.0; 46.6]) showing the lowest CCM percentages. CCM percentages also varied significantly based on the setting where it was performed, ranging from $61.1 \%$ (private clinic) to $86.8 \%$ (endourology and general hospital).

Conclusions: We show that two-thirds of all urological outpatient cases could be completely managed by telemedicine outside of the pandemic. After the pandemic, it will be important to incorporate telemedicine as an alternative for a patient's first or followup visit, especially those with geographical, pathological, and socioeconomic considerations.

\section{Introduction}

With the advances made in personal computer technologies and the expansion of wireless networks worldwide, telecommunication has undergone a drastic revolution in the past decade. These advances offer a plethora of possibilities for telemedicine, but surprisingly, most health systems do not exploit their full potential. This could be due to several barriers, such as a health professionals' unwillingness to change their own practice, reimbursement issues, key legal considerations, or a lack of evidence that telemedicine is an efficient tool for high-quality healthcare. ${ }^{1,2}$ While telemedicine is not appropriate for every type of medical visit, one may imagine several cases in which telemedicine would provide high efficiency, low burden to patients, and maintenance of high-quality care. Recently, with the COVID-19 pandemic, telemedicine has become the cornerstone of medical consultations. Nevertheless, we do not have a complete understanding of its real potential. To determine the ratio of urological appointments that could be fully managed by telemedicine, we conducted a prospective, multisite study involving all urologists practicing in the region of Quebec City, Canada (population of 750000 for primary and secondary urological care and 2 million as a tertiary center). We report the percentage of appointments that were considered by urologists to be completely managed by telemedicine.

\section{Methods}

Between the second and fifth week of the regional confinement period, during which physicians were asked to manage all patients by telemedicine when possible (March 23 to April 16, 2020), every urologist from the Quebec City region was invited to fill out a questionnaire after a phone appointment with their patients (for first or followup visits). They were asked to determine if telemedicine allowed 
either: 1) incomplete case management (ICM) necessitating an in-person visit; 2) complete case management (CCM); or 3) suboptimal case management (SCM), but otherwise adequate during COVID-19 pandemic. The primary objective of this study was to evaluate the rate of CCM by telemedicine in urology.

\section{Results}

During the study period, a total of 1679 cases were completed by telemedicine and analyzed in three different settings: oncology clinic, endourology and general hospital, and urology private clinic (Table 1). All 18 urologists working in the clinic or in the single hospital of the region participated, hence covering all types of consultations performed during that time. The frequency of consultations for nononcological, oncological, suspicion of cancer, or pediatric reasons was $40.9 \%, 37.5 \%, 6.2 \%$, and $8.5 \%$, respectively (7.0\% were not classified by the urologist) (Table 1$)$.

Based on urologists' best opinion, $67.6 \%$ (95\% confidence interval $[\mathrm{Cl}] 65.3 ; 69.8), 27.1 \%(25.0 ; 29.3)$, and $4.4 \%(3.4 ; 5.4)$ of the appointments were classified as CCM, SCM, or ICM, respectively (Table 2$)$. For $1.0 \%(0.6 ; 1.5)$ of consultations, no classification was done.

When analyzing CCM according to the case type, appointment for cancer suspicion had a significantly lower CCM percentage by telemedicine than the oncological and non-oncological appointments $(52.9 \%[42.9 ; 62.8]$ vs. $71.9 \%$ [68.2; 75.3] and 72.7\% [69.2; 76.0], respectively) (Table 2). More complex castration-resistant prostate cancer patients were analyzed separately but showed a CCM ratio of $75.0 \%(47.6 ; 92.7)$, similar to other oncological diseases. For the pediatric population, CCM ratio was $38.0 \%$ (30.0; 46.6), with a significantly lower CCM percentage than that for the adult population $(70.3 \%$ [68.0; 72.6], $\mathrm{p}<0.001)$.

\begin{tabular}{lcc}
\hline Table 1. Appointment characteristics & & \\
\hline \multicolumn{2}{l}{$\mathbf{n}$} & $\%$ \\
\hline Type & & \\
Adult non-oncological & 686 & 40.9 \\
Adult oncological & 629 & 37.5 \\
Adult cancer suspicion ${ }^{1}$ & 104 & 6.2 \\
Pediatric & 142 & 8.5 \\
Not classified & 118 & 7.0 \\
Total & 1679 & 100.0 \\
Setting & & \\
Oncology clinic & 677 & 40.0 \\
Endourology/general hospital & 152 & 9.1 \\
Private clinic & 847 & 50.4 \\
Unknown & 3 & 0.2 \\
Total & 1679 & 100.0 \\
\hline
\end{tabular}

${ }^{1}$ Hematuria, elevated prostate-specific antigen, abnormal digital rectal exam, renal or testicular or vesical mass on imaging.
We also compared CCM according to practice locations. Consultations made from general and endourology hospitals had a higher ratio of CCM $(86.8 \%[80.4 ; 91.8])$ than those performed from urology private clinics or oncology clinics $(61.2 \%$ [57.8; 64.5] and $71.3 \%$ [67.8; 74.7]). Finally, we evaluated inter-physician agreement for CCM between health providers by comparing CCM ratio between the six uro-oncologists. Physicians had significant inter-observer differences in CCM percentages but the inter-observer impact on the decision was modest (c-index of 0.6).

\section{Discussion}

Our study presents important data in favor of the broader use of telemedicine in urology. First, we show that two-thirds $(67.6 \%)$ of urological medical appointments can be managed completely by telemedicine. This number includes all types of appointments for benign or cancerous uropathologies, both in the adult and pediatric populations. Our ratio of CCM is consistent with that reported by Boehm et al in a smaller series of 399 patients from a tertiary center. ${ }^{3}$ In their series, $63.2 \%$ of patients were judged eligible for telemedicine after retrospective chart review by four physicians. Importantly, another recent study reported that direct consultations were reduced from $63 \%$ to $9 \%$ between weeks 1 and 4 after confinement in Italy, meaning that telemedicine indications might be expanded in the context of a pandemic. ${ }^{4}$ To ensure our results would be valid after the pandemic, we asked the participating physicians to judge if the case management by telemedicine would have been optimal (CCM) or suboptimal (SCM) outside of the COVID pandemic. Because telemedicine was considered adequate but suboptimal for $27.1 \%$ of cases, it might still offer the best management in some of these SCM appointments once geographical, pathological, and socioeconomic factors are considered. For instance, we recently reported that uro-pediatric patients travelled, on average, a $69 \mathrm{~km}$ distance one way for a urological visit at our center. ${ }^{5}$ It seems reasonable that many parents would have chosen telemedicine from the $58.5 \%$ of pediatrics appointments judged as SCM by pediatric urologists. This shows that telemedicine probably would be optimal in more than our reported ratio of CCM. In adult cases, cancer suspicion consultations had the lowest CCM ratio $(57.2 \%)$, which is consistent with the study by Luciani and al reporting that for this type of consultation, the majority of patients still required in-person visits even during the peak of the pandemic in Italy. ${ }^{4}$

Our study has some limitations, one being that we focused on the urologist's perspective to determine telemedicine adequacy and we acknowledge that the patient's experience after telemedicine appointment is of prime importance. ${ }^{6}$ In line with this, it is noteworthy that similar studies performed during or outside of the COVID pan- 


\begin{tabular}{|c|c|c|c|c|c|c|c|c|}
\hline & \multicolumn{2}{|c|}{ CCM } & \multicolumn{2}{|c|}{ SCM } & \multicolumn{2}{|c|}{ ICM } & \multicolumn{2}{|c|}{ Not classified } \\
\hline & $\%$ & $95 \% \mathrm{Cl}$ & $\%$ & $95 \% \mathrm{Cl}$ & $\%$ & $95 \% \mathrm{Cl}$ & $\%$ & $95 \% \mathrm{Cl}$ \\
\hline \multicolumn{9}{|l|}{ Type $^{1}$} \\
\hline Adult non-oncological & 72.7 & $(69.2 ; 76.0)$ & 24.2 & $(21.0 ; 27.6)$ & 2.2 & $(1.2 ; 3.6)$ & 0.9 & $(0.3 ; 1.9)$ \\
\hline Adult oncological & 71.9 & $(68.2 ; 75.3)$ & 22.4 & $(19.2 ; 25.9)$ & 5.1 & $(3.5 ; 7.1)$ & 0.6 & $(0.2 ; 1.6)$ \\
\hline Adult cancer suspicion ${ }^{3}$ & 52.9 & $(42.9 ; 62.8)$ & 39.4 & $(30.0 ; 49.5)$ & 5.8 & $(2.2 ; 12.1)$ & 1.9 & $(0.2 ; 6.8)$ \\
\hline Pediatric & 38.0 & $(30.0 ; 46.6)$ & 58.5 & $(49.9 ; 66.7)$ & 0.7 & $(0.0 ; 3.9)$ & 2.8 & $(0.8 ; 7.1)$ \\
\hline Not classified & 63.6 & $(54.2 ; 72.2)$ & 20.3 & $(13.5 ; 28.7)$ & 16.1 & $(10.0 ; 24.0)$ & 0.0 & \\
\hline Total & 67.6 & $(65.3 ; 69.8)$ & 27.1 & $(25.0 ; 29.3)$ & 4.4 & $(3.4 ; 5.4)$ & 1.0 & $(0.6 ; 1.5)$ \\
\hline \multicolumn{9}{|l|}{ Setting $^{2}$} \\
\hline Oncology clinic & 71.3 & $(67.8 ; 74.7)$ & 22.3 & $(19.2 ; 25.6)$ & 4.9 & $(3.4 ; 6.8)$ & 1.5 & $(0.7 ; 2.7)$ \\
\hline Endourology/general hospital & 86.8 & $(80.4 ; 91.8)$ & 9.9 & $(5.6 ; 15.8)$ & 3.3 & $(1.1 ; 7.5)$ & 0.0 & \\
\hline Private clinic & 61.2 & $(57.8 ; 64.5)$ & 34.0 & $(30.8 ; 37.3)$ & 4.1 & $(2.9 ; 5.7)$ & 0.7 & $(0.3 ; 1.5)$ \\
\hline $\begin{array}{l}\text { Quantitative variables are described as freque } \\
\text { comparisons. Statistical analyses were perfor } \\
\text { 3hematuria, elevated prostate-specific antiger } \\
\text { incomplete case management; SCM: subopti }\end{array}$ & percent & tal exam, renal & limits. & mass on imagin & approp & exact tests wer & or cat & $\begin{array}{l}\text { data } \\
\text { <0.001; } \\
\text { rval; ICM: }\end{array}$ \\
\hline
\end{tabular}

demic have shown a satisfaction ratio reaching up to $88 \%$ after telemedical consultations. . $^{3,7}$,

Taken together, the results from this study are complementary and add to other recently performed studies to show that telemedicine can be used safely to manage a majority of urological patients. We showed that telemedicine should have an ongoing role outside the pandemic and that we should be giving serious thought to its continuance.

Competing interests: Dr. Bureau has received speaker honoraria from Pfizer. Dr. Pouliot has been an advisory board member for Amgen, Astellas, Astra Zeneca, Bayer, Janssen, Sanofi, and Tersera; a speakers' bureau member for Astellas and Janssen; and has received payment, honoraria and/ or grants from Amgen, Astellas, Astra Zeneca, Bayer, Janssen, Sanofi, and Tersera. The remaining authors report no competing personal or financial interests related to this work.

This paper has been peer-reviewed

\section{References}

1. Hollander JE, Sites FD. The transition from reimagining to recreating healthcare is now. NEJM Catalyst Innovations Care Delivery 2020;1. https://doi.org/10.1056/CAT.19.1111

2. Ellimoottil C, Skolarus T, Gettman M, et al. Telemedicine in urology: State-of-the-art. Urology. 2016;94:106. https://doi.org/10.1016/j.urology.2016.02.061

3. Boehm K, Ziewers S, Brandt MP, et al. Telemedicine online visits in urology during the COVID19 pandemic — potential, risk factors, and patients' perspective. Eur Urol 2020;78:16-20. https://doi.org/10.1016/i.eururo.2020.04.055

4. Luciani LG, Mattevi D, Cai T, et al. Teleurology in the time of COVID-19 pandemic: Here to stay? Urology 2020;140:4-6. hitps://doi.org/10.1016/j.urology.2020.04.004

5. Otis-Chapados $S$, Coderre K, Bolduc $S$, et al. Evaluating the distance travelled for urological pediatric appointments. Can Urol Assoc J 2019;13:391-4. https://doi.org/10.5489/cuai.5892

6. Chaet D, Clearfield R, Sabin JE, et al. Ethical practice in telehealth and telemedicine. J Gen Intern Med 2017;32:1136-40. https://doi.org/10.1007/s1 1606-017-4082-2

7. Viers BR, Lightner DJ, Rivera ME, et al. Efficiency, satisfaction, and costs for remote video visits following radical prostatectomy: A randomized controlled trial. Eur Urol 2015;68:729-35. https://doi. org/10.1016/i.eururo.2015.04.002

8. Powell RE, Henstenburg JM, Cooper G, et al. Patient perceptions of telehealth primary care video visits. Ann Fam Med 2017;15:225-9. https://doi.org/10.1370/afm.2095

Correspondence: Dr. Frédéric Pouliot, Department of Surgery, Faculty of Medicine, Université Laval, Quebec City, QC, Canada; frederic.pouliot@crchudequebec.ulaval.ca 